\title{
Masquerading as A Stone: An Unusual Cause of Chronic Ureteric Obstruction
}

\author{
(1) Oliver van den Broek Best ${ }^{1}{ }^{2}$, (D) Lequang Vo ${ }^{1}$, (D) Marcus Handmer 1,3 , (D) Fiona Maclean ${ }^{4}, 5$, (1) Pascal Mancuso ${ }^{1,3}$ \\ 'Department of Urology, Liverpool Hospital, Liverpool, Australia \\ 2Sydney Medical School, Faculty of Medicine and Health, Sydney, Australia \\ 3 University of New South Wales Faculty of Medicine, Sydney, Australia \\ ${ }^{4}$ Anatomical Pathology, Douglass Hanly Moir Pathology, Sydney, Australia \\ 5 Macquarie University Faculty of Medicine, Department of Clinical Medicine, Sydney, Australia
}

\begin{abstract}
Amyloidosis is a group of disorders characterized by the extracellular deposition of amorphous, misfolded proteins. Primary localized bladder amyloidosis is a rare form of the disease that usually presents with macroscopic hematuria, irritative lower urinary tract symptoms, or a bladder mass on imaging. Presented herein is a case of a 76-year-old female who presented with sepsis, distal ureteric calculus, and chronic ureteric obstruction without the classical symptoms of bladder amyloidosis. Cystoscopic resection of thickened bladder wall was positive for amyloid deposition with no signs of malignancy.
\end{abstract}

Keywords: Urinary bladder neoplasms, amyloid, ureteral obstruction

\section{Introduction}

Amyloidosis is a benign group of disorders, characterized by extracellular deposition of amorphous, misfolded proteins, known as amyloid fibrils that may have localized or systemic manifestations (1-3). Primary localized bladder amyloidosis (PLBA) is a rare form of the disease, with only a few hundred cases reported in the literature (4). PLBA most commonly presents with painless, macroscopic hematuria, or irritative lower urinary tract symptoms and may mimic bladder carcinoma radiologically and cystoscopically $(2,4,5)$. Radiological findings are generally indeterminate, demonstrating bladder thickening or a discrete mass $(2,6)$. The cystoscopic appearance includes ulcerated lesions, yellow plaques, or irregular thickening, which may suggest malignancy or cystitis $(2,5,6)$. Therefore, PLBA diagnosis relies on histopathological analysis. Congo red stains deposit a salmon pink color, which shows apple-green birefringence on visualization under polarized light $(2,5)$.

\section{Case Report}

Presented herein is a case of a 76-year-old female who presented with a 1-week history of generalized abdominal pain and distension. Her past medical history was significant for hypertension and the use of Chinese and herbal medicines. She was a non-smoker. The initial assessment noted rapid atrial fibrillation with a creatinine of $209 \mathrm{umol} / \mathrm{L}$ (45-90), leukocyte count of $17.4 \times 10^{9}(4-10)$, and a C-reactive protein of $516 \mathrm{mg} / \mathrm{L}$ $(<5)$. A non-contrast computed tomography (CT) scan of the abdomen and pelvis demonstrated a grossly dilated left renal collecting system and ureter (Figure 1). Total cortical loss of the left kidney and a calcific density that measures $6 \times 10 \mathrm{~mm}$ was noted in the region of the distal left ureter. The bladder wall was thickened. A left-sided percutaneous nephrostomy was inserted and purulent fluid was drained. Recovery was complicated by post-procedural Escherichia coli sepsis with rapid atrial fibrillation and hypotension, requiring admission to the intensive care unit for vasopressor support. Her inflammatory markers gradually normalized and creatinine decreased to 96

Correspondence: Oliver van den Broek Best MD, Liverpool Hospital, Clinic of Urology, Sydney Medical School Faculty of Medicine and Health, Department of Urology, Australia

Phone: +61 420997874 E-mail: oliver.best@live.com ORCID-ID: orcid.org/0000-0003-0223-994X

Received: 12.03 .2021

Accepted: 14.05.2021

Cite this article as: Best OVDB, Vo L, Handmer M, Maclean F, Mancuso P. Masquerading as a Stone: An Unusual Cause of Chronic Ureteric Obstruction. J Urol Surg 2021;8(4):303-305.

${ }^{\circledR}$ Copyright 2021 by the Association of Urological Surgery / Journal of Urological Surgery published by Galenos Publishing House. 
umol/L. She was discharged after a short period of rehabilitation with the nephrostomy tube in situ.

A cystoscopy was performed approximately 3 weeks after discharge; the bladder mucosa was abnormal, thickened, and erythematous, suggesting possible high-grade malignancy; however, no papillary lesions or discrete masses were found. The ureteric orifice was not visible before resection. Histopathological analysis of resected mucosa demonstrated extensive, amorphous, and extracellular material, which was positively stained with crystal violet and congo red. On the latter stain, characteristic apple-green birefringence was detected with polarized light, consistent with amyloid deposition (Figure 2). No evidence of dysplasia or malignancy was found. A repeat cystoscopy was successfully performed following the biopsy results and laser lithotripsy of the left distal ureteric calculus. A retrograde ureteric stent was inserted, and then the nephrostomy tube was clamped and subsequently removed. A few months after the removal of the left ureteric stent, the patient was rereferred with worsening renal function. The repeat CT imaging demonstrated bilateral hydronephrosis without ureteric calculi or other causes of obstruction. A cystoscopy demonstrated a similar appearance. A left-sided ureteric stent was inserted; however, despite resection, the right ureteric orifice was not visible. A right-sided percutaneous nephrostomy was then inserted and subsequently removed following the antegrade stent placement. The patient will undergo monitoring and follow-up for ongoing cystoscopic surveillance and ureteric stent exchanges.

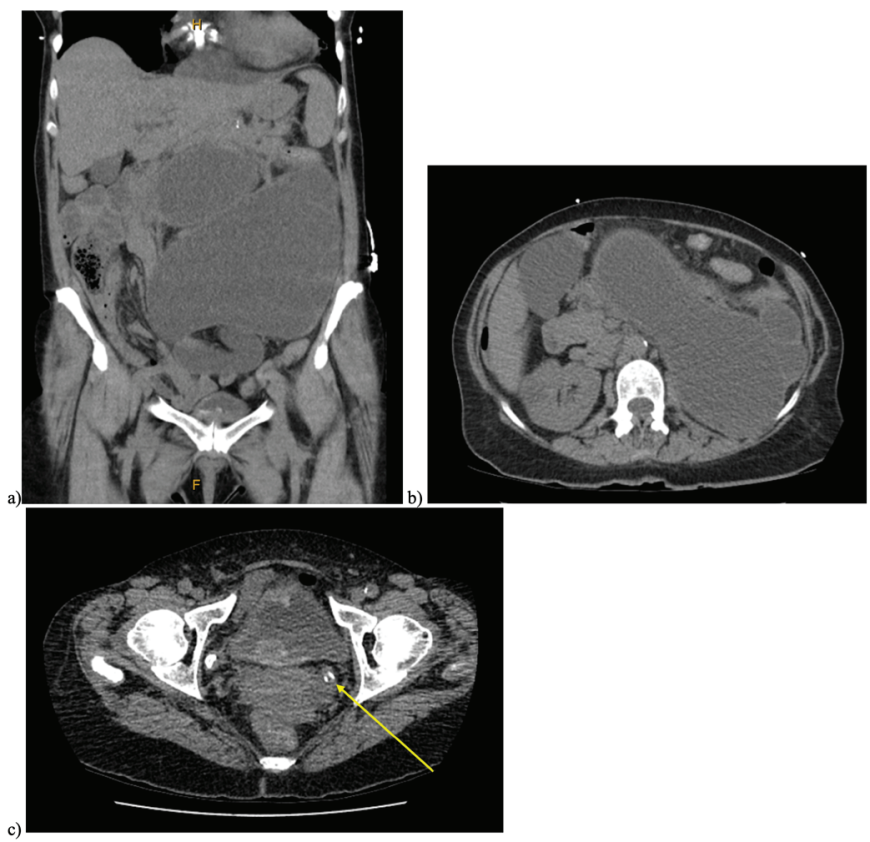

Figure 1. Coronal (a) and axial (b and c) computed tomography images demonstrating massive left-sided hydronephrosis with left renal cortical atrophy, distal left ureteric calculus (yellow arrow), and irregularly thickened bladder wall

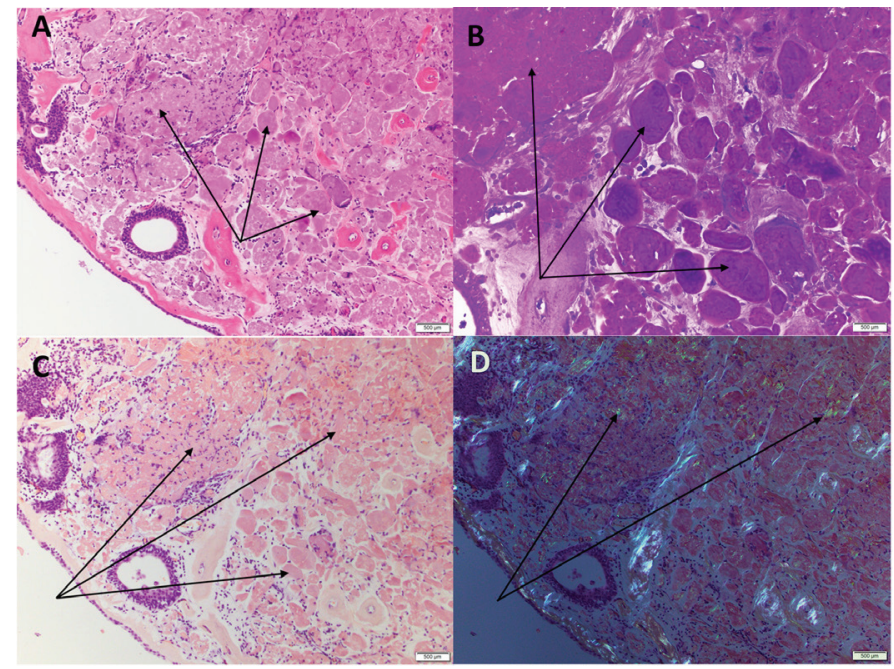

Figure 2. Microscopic bladder biopsy histopathological images at $\times 100$ magnification. Extensive, extracellular, and homogenous amorphous deposits as seen on hematoxylin and eosin stain (A), which was positively stained with crystal violet (B) and confirmed as amyloid deposition with congo red stain (C) and apple-green birefringence under polarized light (D)

\section{Discussion}

The present case is unusual and interesting for several reasons. The patient did not present with the classical symptoms of lower urinary tract symptoms or macroscopic hematuria. She had no signs or symptoms suggestive of systemic amyloidosis. Our patient presented with sepsis due to obstructive uropathy. The PLBA possibly obstructed the left ureteric orifice gradually with subsequent development of hydronephrosis, renal cortical atrophy, and infection.

PLBA has an unknown etiology, but chronic inflammation is suggested to be responsible (2). The mechanism is thought to involve infiltration of the lymphoplasmacytic cells and secretion of abnormal light chain immunoglobulin, which lead to deposition of amyloid fibrils in the bladder mucosa $(2,3)$. An underlying plasma cell neoplasm should be excluded. One possible mechanism of chronic inflammation is the development of a ureteric calculus with subsequent obstructive nephropathy, infection, and development of PLBA. However, this is considered less likely in our case due to the absence of previous renal colic and subsequent development of similar right ureteric obstruction without ureteric calculus.

The main treatment of PLBA is transurethral resection; however, cystectomy or medical therapy have also been used $(3,5,7)$. Given that recurrence rates are as high as 50\%, annual cystoscopic surveillance is recommended $(3,7)$.

In conclusion, this is a very unusual case of PLBA, presenting with infection and chronic ureteric obstruction without lower urinary tract symptoms or macroscopic hematuria. PLBA often mimics bladder cancer but has a benign clinical course. Resection 
with cystoscopic surveillance is the current recommended management.

\section{Ethics}

Informed Consent: Informed consent was obtained.

Peer-review: Internally peer-reviewed.

\section{Authorship Contributions}

Surgical and Medical Practices: M.H., F.M., P.M., Concept: M.H., P.M., Design: O.V.D.B.B., Data Collection or Processing: O.V.D.B.B., L.V., Analysis or Interpretation: O.V.D.B.B., L.V., M.H., F.M., Literature Search: O.V.D.B.B., L.V., Writing: O.V.D.B.B., L.V., M.H., F.M., P.M.

Conflict of Interest: No conflict of interest was declared by the authors.

Financial Disclosure: The authors declared that this study received no financial support.

\section{References}

1. Livneh A, Shtrasburg S, Martin BM, Baniel J, Gal R, Pras M. Light chain amyloidosis of the urinary bladder. A site restricted deposition of an externally produced immunoglobulin. J Clin Pathol 2001;54:920-923.

2. Al-Obaidy KI, Grignon DJ. Primary Amyloidosis of the Genitourinary Tract. Arch Pathol Lab Med 2021;145:699-703.

3. Tirzaman 0, Wahner-Roedler DL, Malek RS, Sebo TJ, Li CY, Kyle RA. Primary localized amyloidosis of the urinary bladder: a case series of 31 patients. Mayo Clin Proc 2000;75:1264-1268.

4. Nesbitt AL, Khan M, Hoag N, Lokan J. Primary bladder amyloidosis as a cause of haematuria. BMJ Case Rep 2018;2018:bcr2018225414.

5. Wilkinson M, Fanning DM, Flood H. Primary bladder amyloidosis. BMJ Case Rep 2011;2011:bcr0520114211.

6. Zhou F, Lee P, Zhou M, Melamed J, Deng FM. Primary localized amyloidosis of the urinary tract frequently mimics neoplasia: a clinicopathologic analysis of 11 cases. Am J Clin Exp Urol 2014;2:71-75.

7. Bardapure M, Namasivayam SK, Rogawski K. Primary localized amylodoisis of bladder: Is there a need for cystoscopic surveillance? Urol Ann 2013;5:309311. 